\title{
Ingenieurkoffer für Experimentalpraktika@home
}

\author{
M. Beitelschmidt ${ }^{1}$, Z. Wang ${ }^{1}$ \\ ${ }^{1}$ Professur für Dynamik und Mechanismentechnik, Institut für Festkörpermechanik, Fakultät Maschinenwesen, TU-Dresden
}

\begin{abstract}
Experimentalpraktika sind ein wesentlicher Baustein im Studium der Ingenieurwissenschaft. Auch wenn virtuelles Lernen (u.a. Virtual-Reality-Experimente) eine gute Unterstützung für die Studierenden darstellt, spielen reell durchgeführte Experimentalpraktika insbesondere im ingenieurwissenschaftlichen Studium eine unverzichtbare, wichtige Rolle. Die experimentellen Versuche verbinden die Theorie und Praxis und motivieren die Lernenden zum Problemlösen.

Dieser Beitrag beschreibt am Beispiel des Projektes „Ingenieurkoffer für Experimentalpraktika@home“, wie Studierende trotz der besonderen Situation infolge der Coronavirus-Pandemie experimentelle Versuche auf dem Fachgebiet Dynamik eigenverantwortlich durchführen können, sowie die ersten Erfahrungen. Die Vor- und Nachteile der Nutzung des mobilen Ingenieurkoffers werden diskutiert und Ansätze zum effektiven Nutzen vorgestellt.
\end{abstract}

Experimental practical courses or practicals are an essential building block in the study of engineering. Even though virtual learning (including virtual reality experiments) has benefits for students, real experiments play an indispensable and important role, especially in engineering studies. The experimental trials connect theory and practice, and motivate learners to solve problems.

Using the example of the project "Ingenieurkoffer für Experimentalpraktika@home" (engineering case for experimental practicals@home), this paper describes how students can conduct experimental trials in the field of dynamics on their own, despite the special circumstances resulting from the coronavirus pandemic, as well as the initial experiences. The advantages and disadvantages of using the mobile engineering suitcase are discussed and approaches on how to effectively use them are presented.

*Corresponding author: zhirong.wang@tu-dresden.de 


\section{Einleitung}

Experimentalpraktika sind ein wesentlicher Baustein im Studium der Ingenieurwissenschaft. Ihre Bedeutung spiegelt sich z.B. in den folgenden Aspekten wider: Verbindung zwischen Theorie und Praxis, Steigerung der Lernmotivation, Erhöhung der Bereitschaft zum Problemlösen, Bereitschaft zur Gruppenarbeit, Validierung einer Berechnung sowie Förderung der Innovationsfähigkeit. Durch Experimentalpraktika werden vorher gelernte theoretische Aussagen durch praktische Beispiele auf ihre Richtigkeit überprüft, und inhaltlich für Studierende lebendiger und einprägsamer veranschaulicht. Dadurch wird den Studierenden der Erwerb der fachspezifischen und fachübergreifenden Kompetenzen vereinfacht. Auch wenn virtuelles Lernen (u.a. Virtual-Reality-Experimente) eine gute Unterstützung für die Studierenden darstellt, spielen reell durchgeführte Experimentalpraktika, insbesondere im ingenieurwissenschaftlichen Studium, eine unverzichtbare, wichtige Rolle.

Das Fachgebiet Dynamik (z.B. Schwingungen, Akustik) wird von Studierenden als interessant, aber zugleich schwierig empfunden. Das liegt häufig im Fehlen des sogenannten "dynamischen Gefühls" zur Schwingung und Akustik. Dieses Gefühl muss erst durch kognitives Lernen gebildet und entwickelt werden. Um für Studierende die Grundlagen von Schwingungen besser und anschaulicher zu machen, hat die Professur Dynamik und Mechanismentechnik sieben "Ingenieurkoffer für Schall- und Schwingungsanalyse" beschafft. Im nachfolgenden Beitrag soll ein kurzer Überblick über die Anwendungsmöglichkeit des Koffers gegeben sowie erste Erfahrungen beim Experimentalpraktika@home und Ansätze zum effektiven Nutzen dargestellt werden.

\section{Konzept des Ingenieurkoffers}

Die Messkoffer ermöglichen die Durchführung einfacher Schwingungs- und Akustikmessungen. Diese können an Maschinen, Fahrzeugen oder Strukturen durchgeführt werden. Mit einem Nutzfrequenz-Umfang des Datenaufzeichnungsgerätes von bis zu $20 \mathrm{kHz}$ ist der komplette Schwingungs- und Akustik-Frequenzbereich abgedeckt. Er soll insbesondere
Studierenden die Möglichkeit eröffnen, durch selbst durchgeführte Messungen den Umgang mit professioneller Messtechnik zu erlernen, eine Finite-Elemente-Methode- (FEM-)Berechnung zu validieren und besseres Verständnisse zu Material- und Berechnungsmodellen zu gewinnen. Somit erhalten die Studierenden die Gelegenheit, ihre fachlichen und experimentellen Fähigkeiten zu vertiefen und zu erweitern.

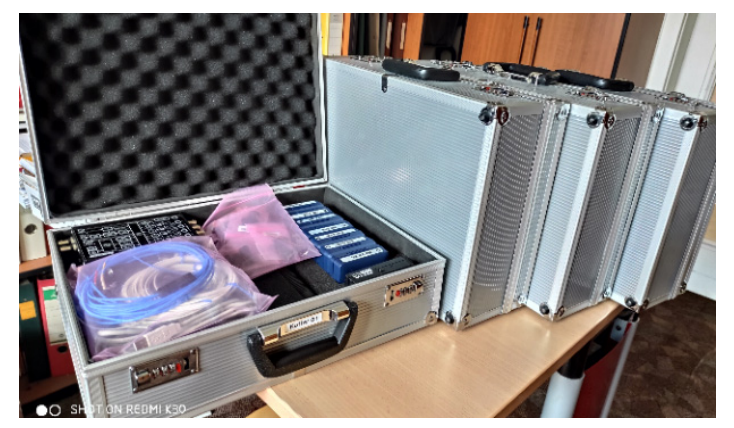

Abb.1: Ingenieurkoffer

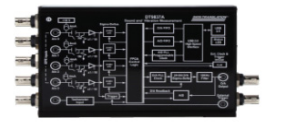

Datenaufzeichnungsgerät

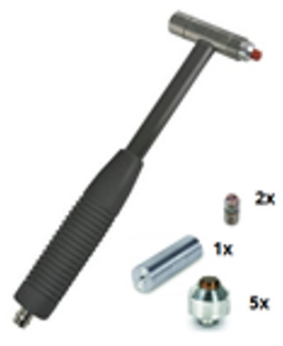

Impulshammer mit Zubehör

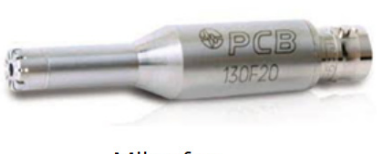

Mikrofon
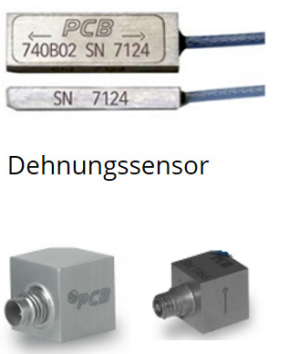

Beschleunigungsaufnehmer
Abb.2: Komponenten im Ingenieurkoffer

Zentrales Bauteil des Koffers ist ein 4-kanaliges Datenaufzeichnungsgerät, das auch Anregungssignale erzeugen kann. Um mechanische Schwingungen messen zu können, sind zwei einachsige und ein dreiachsiger piezoelektrischer Beschleunigungsaufnehmer vorhanden. Dehnungen, die ebenfalls als Messgrößen für mechanische Schwingungen dienen können, können mit den beigefügten Dehnungssensoren gemessen werden. Zwei Mikrofone ermöglichen schließlich die Messung von Luftschall, der häufig als Begleiter mechanischer Strukturschwingungen auftritt. Alle 
Sensoren (inkl. Mikrofone) sind piezoelektrische Sensoren mit eingebauter Elektronik, sogenannte IEPE (Integrated Electronics Piezo Electric) oder ICP (Integrated Circuit Piezoelectric) Sensoren. Dank der integrierten Signalwandlung sind solche Sensoren einfacher in der Handhabung und unempfindlicher gegenüber elektrischen Störungen.

Ein Impulshammer mit integriertem Kraftsensor kann zur kontrollierten Anregung von Schwingungen eingesetzt werden.

Das PC-gestützte Datenaufzeichnungsgerät wird mittels USB an einen Rechner angeschlossen. Eine zusätzliche Signalkonditionierung ist für den Einsatz der gängigen Sensorik nicht erforderlich. Mit der zugehörigen Software kann das Gerät dazu verwendet werden, Messwerte aufzuzeichnen und anzuzeigen, sowie auf der Festplatte des Rechners abzuspeichern. Im Frequenzanalyse-Modus der Software können Analysen wie das Spektrum, das Autospektrum, die spektrale Leistungsdichte, eine Fensterbewertung sowie eine Digital-Filterung durchgeführt werden. Im erweiterten Modus können Spektralanalysen über zwei Signale durchgeführt werden, wie z.B. die Bestimmung von Übertragungsfunktionen, Kreuzspektren sowie Kohärenzfunktionen. Das Gerät kann auch unter allen gängigen Messtechnikapplikationen wie LabVIEW, Matlab, Dasylab oder auch mit Visual Studio eingesetzt werden. Insbesondere die Programmierbarkeit mit MATLAB sowie gängiger Programmiersprache wie .NET und Python eröffnet den Studierenden die Möglichkeit, Mess- und Analysenaufgaben zu kombinieren und die Erkenntnisse über Hardware, Messvorgang und Datenverarbeitung zu vertiefen und zu erweitern. Nicht zuletzt schafft es dem Benutzer die Möglichkeit, neue Anwendungen zu entwickeln und somit Innovationen zu fördern.

Da mehrere Datenaufzeichnungsgeräte miteinander koppelbar sind, können durch die Zusammenführung mehrerer Messkoffer auch komplexere Messungen durchgeführt werden.

\section{Anwendung in der Lehre}

In Sommersemester 2020 konnten die Studierenden in der Lehrveranstaltung "Experimen- telle Modalanalyse" zum ersten Mal die Ingenieurkoffer ausleihen und zu Hause damit experimentieren. Zuerst wurde das theoretische Wissen der Schwingungsanalyse und Modalanalyse durch online-Vorlesungen vermittelt. Die Studierenden konnten die Lernstoffe verstehen und die Theorie durch Übungen und Diskussionen in Fragestunden verinnerlichen. Das normalerweise vorlesungsbegleitende experimentelle Praktikum konnte jedoch wegen des Verbotes der Präsenzveranstaltungen nicht vor Ort stattfinden. Das sollte durch Ausgabe der Koffer an die Studierenden kompensiert werden.

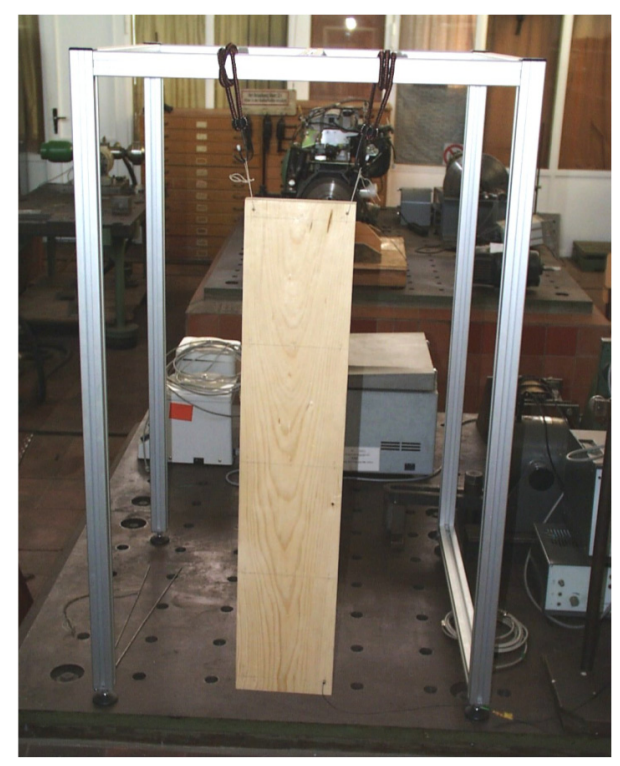

a) Messobjekt

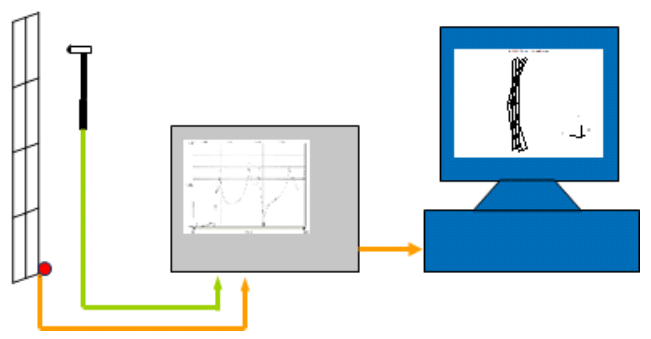

b) Messkette

Abb. 3: Messaufbau zur Modalanalyse einer Holzplatte a) Messobjekt b) Prinzipieller Messaufbau

Die Studierenden lernten dadurch die praktische Vorgehensweise kennen. Sie durften die Messtechnik wie z.B. verschiedene Messsensoren, Anregungsmittel (z.B. Modalhammer unterschiedlicher Größen 5 g - 10 kg, Schwingungserreger verschiedener Typen) in die Hand nehmen, das Messobjekt anfassen und 
die Schwingungen hautnah fühlen. Das erleichtert das Verstehen der theoretischen Grundlagen sowie die Entwicklung eines „dynamischen Gefühls".

Die experimentelle Modalanalyse hat die Aufgabe, aus versuchstechnisch gewonnenen Daten die sogenannten Modalparameter wie z.B. Eigenfrequenzen, Eigendämpfungen sowie Eigenschwingformen einer Struktur zu ermitteln. Die wesentlichen Ziele der experimentellen Modalanalyse sind z.B.:

- Erkennen und Vermeiden von mechanisch oder akustisch störendenden Resonanzen

- Vergleich der Ergebnisse mit dem berechneten Schwingungsverhalten aus numerischen Simulationen, wie z. B. der Finite-Elemente-Methode (FEM), um die in der Berechnung notwendigen Annahmen über Geometrie, Materialparameter, Berechnungsmodell usw. zu überprüfen, und wenn notwendig, zu korrigieren, bis eine zufriedenstellende Übereinstimmung mit den experimentellen Resultaten erzielt wird.

- Ableiten konstruktiver Maßnahmen zur Veränderung von Eigenfrequenzen, wenn Erregerfrequenzen nicht zu beeinflussen sind.

In der zweiten Präsenzveranstaltung wurde ein etwas komplizierteres Messobjekt analysiert. Dabei sollen die möglichen Resonanzfrequenzen eines Blockfundaments (Abb. 4) sowie deren Eigenschwingungsformen ermittelt werden. Diese Aufgabe hat hohe praktische Bedeutung, da Fundamentschwingungen großen Einfluss auf die Präzision und Lebensdauer von Maschinen sowie das Wohlbefinden eines Bedieners haben. Auf noch kompliziertere Messobjekte (Autokarosserie und Straßenbahnwaggon) wurde in diesem Semester verzichtet, da eine Versuchsdurchführung wegen der geltenden Abstands- und Hygieneregeln unmöglich war.

Nach den zwei einführenden Versuchen erfolgte noch einmal zusammenfassend die Einweisung in den Ingenieurkoffer. Dazu gehörte die Erläuterung der Kalibrierdokumente, der
Aufbau der Messkette, die Softwareinstallation, die Einstellung/Bedienung der Messsoftware bis zur kompletten Messung. Sechs Studierende bekamen je einen Ingenieurkoffer ausgehändigt.

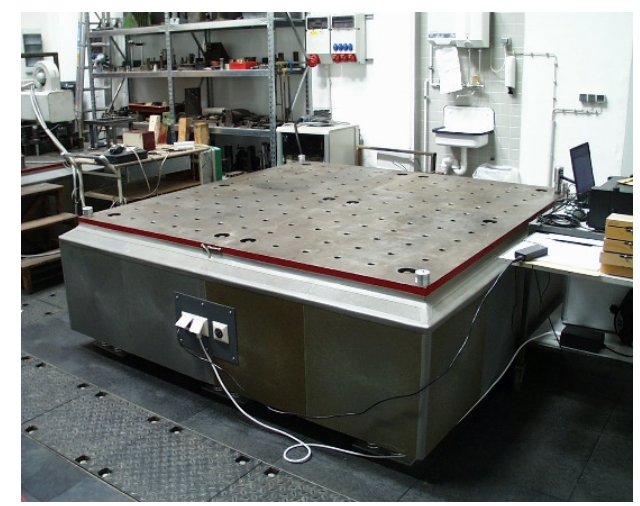

Abb.4: Ermittlung der Eigenfrequenz des Blockfundaments

Mit dem extra für unsere Studierende verfassten Handbuch zum Ingenieurkoffer wurde innen noch umfangreiches Lernmaterial zum Nachschlagen und Selbststudium zur Verfügung gestellt. Das Handbuch beginnt mit einer Einführung in die Messtechnik, insbesondere die Messtechnik im Ingenieurwesen sowie Praxistipps zur Messtechnik. Nach der detaillierten Beschreibung des Kofferinhaltes wird die Nutzungsmöglichkeit der Hard- und Software vorgestellt. Besonders hilfreich für die Studierenden sind die ausführlichen Schritt für Schritt Anleitungen für verschiedene Nutzungsszenarien, wie z.B. Aufzeichnung des Zeitverlaufs von Schwingungsgrößen, Nutzung von Messtrigger, Ad-hoc Frequenzanalyse, Bestimmung der Übertragungs- bzw. Kohärenzfunktion, Steuerung der Anregungssignale sowie Auswertung und Interpretation der Messergebnisse usw.

Mit dem mobilen Ingenieurkoffer und dem theoretischen Grundwissen sollen die Studierenden in die Lage versetzt werden, eigenständig einen selbst gewählten Versuch im Bereich Akustik und Schwingungstechnik zu konzeptionieren, vorzubereiten und durchzuführen. Das Ziel ist es, die experimentellen Fähigkeiten, wie die Konzeption, Planung und Aufnahme von Messreihen, die Plausibilitätskontrolle und Auswertung experimenteller Daten im Rah- 
men der Aufgabenbearbeitung zu fördern. Zugleich wird fachinhaltliches Wissen durch Messungen, Messwertverarbeitung und Ergebnisinterpretation vertieft.

Die Studierenden hatten für ihren Versuch 2-3 Wochen Zeit, danach sollte ein Untersuchungsbericht eingereicht werden.

\section{Betreuungskonzept}

Das Home-Praktikum erfordert ein hohes Maß an Selbstorganisation und Selbstdisziplin der Studierenden. Aber es bedeutet keineswegs, dass die Studierenden für das Home-Praktikum ganz auf sich allein gestellt sind. Für die Konzeption, Vorbereitung sowie Durchführung einzelner Versuche steht den Studierenden eine vielseitige Betreuung zur Verfügung. Speziell angepasste Lernmaterialien werden zur Verfügung gestellt. Außerdem ermöglicht die Digitalisierung ein multimediales Betreuungskonzept, das unter anderem aus den Elementen Foren, Chats, Lernvideos, Videokonferenz usw. besteht. Außerhalb der Präsenzveranstaltung können Studierende mit dem Dozenten oder Betreuer in Kontakt treten, um die Lernmaterialien, Wissenslücken, fachliche Fragen oder auftretende Probleme zu besprechen. Die Kommunikation erfolgt in der Regel via OPAL-Foren, Chats, E-Mail, oder auch telefonisch. Je nach der Dringlichkeit des aufgetretenen Problems werden die Fragen via E-Mail, Foren beantwortet, oder dafür Video-Konferenzen organisiert. Durch (asynchrone) Diskussionen und/oder Video-Konferenzen werden Fragen und Probleme erkannt, Lösungswege gezeigt und die Arbeitsprozesse reflektiert.
Einen Koffer behielt der Betreuer, um eventuelle technische Probleme der Studierenden nachvollziehen und beim online-Support Schritt für Schritt mitmachen zu können.

\section{Ergebnisse}

Einer der sechs Studierenden gab zu, dass er mit dem Koffer nichts gemacht hat. Er begründete es damit, dass er sich dieses Semester keine Zeit dafür nehmen will, da er erfahren habe, dass er gemäß der Prüfungsordnung keine Leistungspunkte durch dieses Fach erzielen kann.

Drei Studierende gaben an, mit der Messtechnik entsprechende Testobjekte untersucht zu haben, jedoch wollten sie aus zeitlichen Gründen kein Versuchsprotokoll anfertigen. Sie berichteten mündlich bei einem Präsenztermin über ihr Versuchsobjekt sowie gesammelte Erfahrungen. Die schließlich von den verbleibenden zwei Studierenden abgegebenen Protokolle sind erfreulicherweise von hoher Qualität. Beide Studenten hatten sich als Testobjekt einen kleinen Tisch ausgesucht.

In den Versuchsprotokollen wurde das Versuchskonzept und der Versuchsaufbau ausführlich dokumentiert, die erzielten Messergebnisse interpretiert und eine Fehlerbetrachtung vorgenommen. Zusätzlich haben die Studierenden Ihre Messergebnisse noch freiwillig mit FEM-Simulationen verglichen, um das Simulationsmodell zu verifizieren und zu validieren (Abb. 5).

Der Vergleich der experimentell ermittelten Modalparameter mit den Berechnungen zeigt
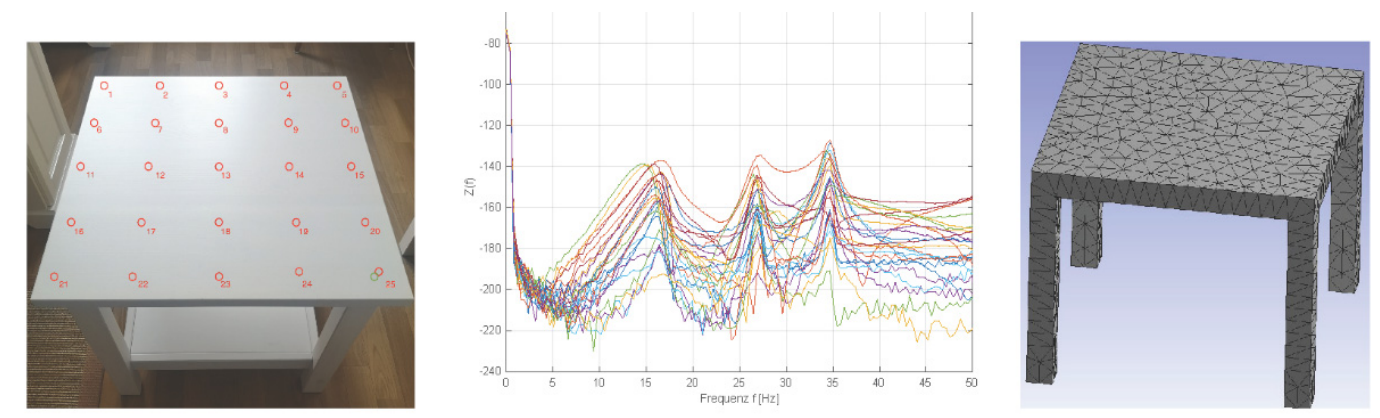

Abb. 5: (links) Messobjekt und Messpunkte, (Mitte) gemessene Frequenzgänge, (rechts) CAD/FEM-Modell (Beleg des Studenten L. Hollas und V. Scholz) 
auf, dass die Berechnung mit dem Messergebnis nicht ganz übereinstimmt. Deswegen wurde eine Ursachensuche und Diskussion zu verschiedenen Fragen zur Berechnung sowie dem Versuch vorgenommen, wie z.B.

- Sind die Ergebnisse plausibel? Die Plausibilitätskontrolle ist ein Grundwerkzeug, mit dem Berechnungs- oder Messfehler bzw. Interpretationsprobleme aufgedeckt werden können.

- Sind das FEM-Modell, die Materialparameter und Geometrie korrekt? Wurden bei der Modellierung die besonderen Eigenschaften des Holzstoffes (Kunststoff, Leichtbaustoff, ...) ausreichend berücksichtigt? Ist demzufolge meine FEM-Berechnung vertrauenswürdig?

- Sind die Randbedingungen, z.B. Lagerung des Versuchsobjektes geeignet?

- Sind die Erregungskräfte beim Schwingungsversuch ausreichend und geeignet?

- Sind die Komponenten des Messsystems (Aufnehmer, Shaker, Modalhammer, Datenerfassungsgerät, Verstärker, Kabel usw.) für den Versuch aufeinander abgestimmt?

- Stimmt der Versuchsaufbau und die Messkette?

- Werden die Messdaten richtig ausgewertet?

Bei einem Feedbackgespräch gaben die Studierenden an, durch das Experimentalpraktikum ihre Kompetenz hinsichtlich wissenschaftlichen Denkens und empirischen Arbeitens erweitert zu haben.

\section{Vor- und Nachteile}

Die durch die Corona-Pandemie bedingte Verlagerung des universitären Lehrbetriebs vom Hörsaal in den digitalen Raum sowie die strengen Hygiene-Regeln führen dazu, dass die Praktika in der klassischen Form nicht durchführbar sind. Auch wenn das Home-Praktikum aus der Not entstanden ist, schienen aber viele Vorteile für solche Praktika@home zu sprechen:

- Flexibilität in zeitlicher und räumlicher Hinsicht, da der Wissenserwerb nicht an fixe
Termine gebunden, sondern zu jedem beliebigen Zeitpunkt möglich ist. Dies ermöglicht ein Lernen gemäß dem eigenen Rhythmus, ohne sich mit Dozenten oder anderen Teilnehmern abstimmen zu müssen.

- Freie Wahl der Lerngeschwindigkeit und Lernintensität: Die Studierenden können entscheiden, welche und wie viele Informationen sie jeweils aufnehmen möchten, das heißt, wie schnell und intensiv sie lernen wollen, - ob sie beispielweise ein bestimmtes Experiment ganz oder nur zum Teil wiederholen möchten.

- Abrufung von Zusatzinformationen je nach individuellen Bedürfnissen und Ansprüchen, ohne für sie uninteressante Informationen aufnehmen zu müssen.

- Digitalisierung ermöglicht eine gute Kommunikation mit Betreuer und Gruppenarbeit zwischen den Mitstudierenden.

Ein weiterer wesentlicher Vorteil der HomePraktika ist, dass die Messobjekte in der Regel authentisch und realitätsnah sind, (wie z.B. hier ein kleiner Tisch). Andere mögliche Messobjekte wären z.B. das Schwingungsverhalten des eigenen PKW oder die Fußbodenbelastung durch eine schleudernde Waschmaschine. Die Motivation für die Praktika würde steigen. Die Studierenden bekommen dadurch Freiraum zur Verwirklichung ihrer eigenen kreativen Ideen. Aus den berichteten Ergebnissen ist zu erkennen, dass die meisten Studierenden für das Home-Praktikum sehr motiviert waren, obwohl die Qualität der abgegebenen Protokolle keinen direkten Einfluss auf die Note hat.

Dennoch zeigt unser Pilotversuch zumindest teilweise eine ernüchternde Bilanz. Die angebotene Möglichkeit führt nicht zwangsläufig zu einer besseren Lernkompetenz und zu mehr Wissen. Die Durchführung experimenteller Versuche ohne unmittelbare Hilfeleistung von Dozenten mag die Selbstständigkeit der Studierenden fördern, kann jedoch für andere, wegen der fehlenden Möglichkeit für unmittelbare Rückfragen, demotivierend sein. Missdeutungen und Fehler werden selten aufgedeckt und verhindern im schlimmsten Fall den Lernerfolg. Zudem werden Unsicherheiten und Irritationen nicht aufgefangen und können darum auf lange Sicht zu Frustration füh- 
ren. Auch kreative Problemlösungsdynamiken, wie sie innerhalb von Präsenzveranstaltungen häufig entstehen, kommen auf diese Weise nicht zustande.

Noch ein sehr allgemeines Problem ist: Nicht alle Studierenden sind bereit, ihr Lernen in die eigene Hand zu nehmen. Das Lernen zu Hause verlangt ein hohes Maß an Selbstdisziplin, das nicht jeder beziehungsweise jede aufzubringen in der Lage ist [2].

Die hier genannten Probleme bedeuten natürlich nicht, dass die zuvor dargestellten Vorteile nicht gültig seien. Es ist uns klar, dass das Home-Praktikum ergänzt werden muss: Es muss mit Präsenzlehre oder mindestens Video-Konferenzen gekoppelt werden. Die Kombination von Home-Praktika und verschiedenen Videokonferenzen/Präsenzlehre kann auch die für das Selbstlernen typischen didaktischen Probleme kompensieren. Auch nach der Pandemie können die Home-Praktika ein sinnvolles und erfolgsversprechendes Zusatzangebot darstellen, jedoch kein Ersatz für die Vorort-Praktika sein.

Ein weiteres Problem für Home-Praktika ist der erhöhte Verschleiß der Messtechnik, damit verbunden sind die erhöhten Betriebskosten und die Ersatzbeschaffung. Sowohl die Messtechnik wie Sensoren, als auch das Zubehör wie diverse Kabel und Adapter sind empfindlich gegen unsachgemäße mechanische Belastung. Es wurden bereits erste kleine Schäden festgestellt. Die Gründe dafür können vielseitig sein. Einerseits sind die einzelnen Bestandteile im Ingenieurkoffer nicht robust und strapazierfähig gebaut, anderseits fehlt den Studierenden trotz ausführlicher Einweisung noch die Erfahrung, mit empfindlicher Messtechnik umzugehen. Das Sammeln dieser Erfahrung ist jedoch gerade eines der Lernziele eines Praktikums.

\section{Schlussfolgerung}

Der erste Pilotversuch für Home-Praktika hat wichtige Erkenntnisse für die zukünftige Durchführung geliefert. Home-Praktika können motivierten Studierenden eine große Chance bieten, ihr erworbenes Wissen anzuwenden und fächerübergreifend umzusetzen. Zusätzlich stärken Home-Praktika ihre Selbstständigkeit und Handlungsorientierung. Die
Selbstdisziplin, Lernorganisation, Zeitmanagement und nicht zuletzt die Motivation der Studierenden beeinflussen das Ergebnis der Home-Praktika, wobei die Motivation das Wichtigste für Erfolg ist. Es ist entscheidend, Wege zu finden, um die Motivation zu steigern.

Zur Steigerung der Motivation wurden verschiedene didaktische Methoden eingesetzt, zum Beispiel wurden im Rahmen der Lehrveranstaltung den Studierenden die Gelegenheit gegeben, ihre Aufgaben aus eigener Kraft zu bewältigen und damit ihr eigenes Handeln als wirksam und effektiv wahrzunehmen (Kompetenzerlebnis). Sie bekamen Freiraum, selber zu entscheiden, welches Testobjekt sie untersuchen möchten, in welchem Umfang sie sich mit dem Thema auch fachübergreifend beschäftigen, und ihre Versuche so zu gestalten, dass ein Optimum im Sinne der Aufgabenerledigung erzielt wird (Autonomie), und das Gefühl, dass sie etwas sehr Praktisches und Wichtiges zu tun haben (Bedeutsamkeit) [1]. Dafür ist eine strukturierte Planung der Lehrveranstaltung, der Aufgabenstellung und der Lernziele notwendig. Die Vorlesung und auch die theoretische Übung müssen mit der Aufgabenstellung so abgestimmt werden, dass die Aufgaben für Home-Praktika herausfordernd und gleichzeitig zu bewältigen sind. Die erforderliche Vorbereitung, bzw. eventuell bei der Versuchs-Durchführung auftretende Probleme, sollten vorher in einer Präsenzrunde diskutiert werden, sodass die Studierenden bei HomePraktika nicht überfordert sind und von sich aus Freude an den Aufgaben haben. Außerdem sollten sie genau wissen, welche Inhalte von Bedeutung sind, welche Lernziele bei jedem Praktikum zu erreichen sind (Transparenz). Für technische und methodische Fragen soll eine zeitnahe (online-) Unterstützung gewährleistet werden.

Auch die zusätzliche Aktivierung extrinsischer Motivation, d.h. Motivation aufbauend auf Anreiz von außen, ist sinnvoll, da nicht alle Studierenden die intrinsische Motivation für ihre Arbeit mitbringen. Das gilt deshalb, weil die Entscheidung für das Fach häufig in bestimmtem Ausmaß durch extrinsische Faktoren angeregt ist, da die Lehrveranstaltung „Experimentelle Modalanalyse" eine sehr hohe Praxisrelevanz besitzt und ein wesentliches Standbein gegenüber der rechnerischen Simulation darstellt. 
Ein möglicher Anreiz wäre es, zusätzliche Bonus-Punkte für die Praktika in der Modulbewertung zu verankern. Die Bonusregelung bietet den Studierenden einen Anreiz zum kontinuierlichen Lernen im Semester und fördert somit ein besseres Lernen. So können sich Studierende z.B. durch benotete Hausaufgaben oder Experimente ein ,Extra' zur anstehenden Prüfung dazuverdienen. Das muss aber in der Prüfungsordnung entsprechend vorgesehen werden.

\section{Ausblick}

Die ersten geschilderten Eindrücke machen deutlich, dass Experimentalpraktika@home einen besonderen Mehrwert für den Kompetenzerwerb bringen. In Zukunft werden die Ingenieurkoffer zusätzlich zum Fach „Experimentelle Modalanalyse" auch in weiteren Lehrveranstaltungen wie z.B. Messwertverarbeitung und Diagnostik, Schwingungslehre eingesetzt.

Mit dem Inkrafttreten der neuen Studien- und Prüfungsordnungen werden ab Sommersemester 2021 auch Studierende der Vertiefung "Simulationsmethoden des Maschinenbaus" und "Allgemeiner und Konstruktiver Maschinenbau" die oben genannten Lehrveranstaltungen besuchen, damit steigt der Bedarf an Ingenieurkoffern. Die Professur Dynamik und Mechanismentechnik bemüht sich, weitere Koffer und Software anzuschaffen, sowie Mittel für entsprechende Betriebskosten bereitzustellen.

\section{Danksagungen}

Die sieben "Ingenieurkoffer für Schall- und Schwingungsanalyse" wurden mit ZUK-Mitteln zur Unterstützung der Lehre finanziert.

\section{Literatur}

[1] Egger, Rudolf; Merkt, Marianne: Lernwelt Universität: Entwicklung von Lehrkompetenz in der Hochschullehre, Springer Verlag, 2012.

[2] Kuhlen, Rainer: Hypertext. Ein nichtlineares Medium zwischen Buch und Wissensbank, Berlin SpringerVerlag, 1991 